\title{
« Eau et territoires : quelles priorités pour la recherche? »
}

\section{Compte rendu de colloque (Lyon, 9-10 janvier 2006)}

\author{
Alain Pavé \\ Biométricien, UPS CNRS-Guyane, 16 avenue André Aron, 97300 Cayenne, Guyane
}

Ce colloque était organisé par le Cemagref. Le thème en était précisé par le sous-titre: «Vers une nouvelle approche de la gestion de la ressource, du risque et des espaces ». L'objectif était d'arriver à des conclusions susceptibles d'aboutir à un appel d'offres proposable aux organismes de recherche et à l'Agence nationale de la recherche.

Bien qu'on puisse s'en étonner, coupler les questions de l'eau avec celle des territoires n'est pas une pratique courante, ni chez les scientifiques ni chez les gestionnaires. Chez les scientifiques, on peine à promouvoir des recherches interdisciplinaires, l'eau dans notre environnement reste principalement une affaire d'hydrologue. Chez les gestionnaires, le partage des tâches conduit à découper l'action en secteurs suivant l'objet à gérer : l'eau, les territoires, voire leurs composantes (systèmes ruraux, agricoles ou forestiers, espaces naturels, systèmes urbains et périurbains, réseaux de transports, etc.), et la nature du problème (eau comme ressource, eau comme vecteur, eau comme facteur de risques). L'échelle reste souvent le bassin versant.

L'idée de ce colloque était donc de promouvoir une approche interdisciplinaire sur la thématique " eau et territoires », pour acquérir des connaissances nouvelles grâce à l'étude de ce couplage, mais aussi de privilégier une recherche qui puisse se traduire en termes de gestion. En effet, nous savons, depuis l'invention des sciences pour l'ingénieur, que le passage à la technique et à sa mise en œuvre demande une recherche spécifique. De plus, on ne s'étonnera pas que les aspects méthodologiques et conceptuels, cruciaux dans ce type d'approche, aient été mis en avant.

Du point de vue scientifique, il s'agissait non seulement de faire collaborer chercheurs et gestionnaires, mais, dans chacun des ensembles, de promouvoir des approches

Auteur correspondant : alain.pave@cnrs-dir.fr réunissant : du côté des chercheurs, les sciences de la nature, les sciences de l'homme et de la société, ainsi que les sciences pour l'ingénieur; du côté des gestionnaires, les différentes catégories et les échelles multiples (gestion de l'eau, gestion des territoires et ingénierie de l'aménagement et de l'équipement).

À l'issue de cette réunion, on peut dire que le pari a été gagné, même si le sentiment persiste qu'il reste beaucoup à faire pour vaincre les inerties de tous ordres, notamment celles de la communauté scientifique. À ce propos, il est intéressant de noter que cette initiative vient d'un " petit organisme ». La petite taille peut être un handicap, car elle limite le champ de compétences. Mais c'est aussi un avantage, car elle permet d'éviter les lourdeurs et de créer les conditions favorables à l'initiative et à l'inventivité. C'est ce qu'a réussi le Cemagref.

L'investissement mis dans la préparation et l'organisation du colloque a rendu le travail très efficace. Cela a conduit à un ensemble de conclusions opérationnelles. Les organisateurs avaient rédigé un texte de cadrage précis destiné à lancer et, si possible, à orienter les débats, en offrant la possibilité $\mathrm{d}^{\prime}$ intervenir y compris aux participants qui étaient en désaccord avec ce texte. Les axes considérés comme prioritaires étaient : fonctionnement des systèmes biophysiques et spatialisation; systèmes d'information environnementaux; impacts réciproques entre sociétés et systèmes biophysiques; outils pour le pilotage des systèmes environnementaux; usages, gouvernance et développement territorial. On peut certes discuter un tel plan, mais il a l'avantage d'exister et, donc, de permettre le démarrage d'une discussion, qu'on l'accepte ou non.

Après que Claudine Schmidt-Lainé (Cemagref) eut présenté les objectifs de la rencontre, une série d'exposés introductifs en ont balisé les contours. Pour sa part, Laurent Mermet (Engref) a critiqué le choix du bassin 
versant comme échelle presque unique de recherche et d'action. Il a illustré au contraire la diversité des approches sectorielles possibles et même nécessaires, aussi bien du point de vue des gestionnaires que des scientifiques, regrettant leur «étanchéité » les unes par rapport aux autres. Il a ensuite proposé des démarches intégrées et systémiques, en insistant sur le rôle du modèle, mais en pointant aussi ses limites et le rôle dogmatique que l'on pourrait lui faire jouer. Paul Caseau (Académie des technologies) a repris le schéma en trois pôles : naturesociété-économie, dont il a supervisé la réalisation comme support de réflexion et qu'il a présenté dans le Rapport sur la science et la technologie (RST) $n^{0} 15^{1}$. Il a mis l'accent sur la question du choix des échelles pertinentes, car c'est un choix souvent conflictuel. Il a conclu en évoquant les outils et les méthodes utilisables (observatoires, ensembles de données, modèles, simulation). Maurice Héral (Ifremer) a présenté le problème posé par le partage des compétences et des territoires en s'appuyant sur l'exemple de la bande côtière et des estuaires (ce qui revient au marin et ce qui revient au continental) et, lui aussi, a prôné une approche intégrée, thématique, plutôt que sectorielle, institutionnelle et géographique. Paola Rizzi (Université de Sassari, Sardaigne) a montré l'importance d'approches conceptuelles et l'intérêt du recours à des notions fortes, comme celle de résilience, pour les systèmes naturels, certes, mais aussi pour les systèmes sociaux. Henri Tardieux (Compagnie d'aménagement des coteaux de Gascogne, CACG) a fort justement insisté sur l'importance des aménagements ( Aménager n'est pas une injure! »). Cela a donné l'occasion de parler de la composante ingénierie, trop souvent mise de côté dans les diverses réflexions. Les attentes et les perspectives en termes de gestion ont été exposées par Jacques Theys (ministère de l'Équipement).

La réflexion était organisée autour de cinq ateliers : 1. Bassins versants terrestres et côtiers, milieux et développement territorial ; 2 . Natures, cultures et paysages ; 3 . Tensions, partages et conflits ; 4 . La ville et son environnement ; 5 . Climat, phénomènes extrêmes. La synthèse des travaux des ateliers a été présentée par Bernard Barraqué, président du comité scientifique du colloque, qui a mis en avant le point de vue économique et gestionnaire qu'introduit précisément la dimension territoriale.

Les discussions des ateliers ont été nourries. Ceux-ci avaient des notes de cadrage à suivre. Les règles proposées ont été appliquées plus ou moins scrupuleusement, sauf un cas d'opposition presque complète, mais assorti d'explications et d'une contre-proposition. Il n'est pas possible d'entrer dans le détail de ces travaux. Mais on peut en extraire un axe de réflexion révélateur. En effet, deux conceptions semblent s'affronter dans le débat. Prenons

\footnotetext{
1 Académie des sciences, Caseau, P. [animateur], 2003. Études sur l'environnement : de l'échelle du territoire à celle du continent, Paris, Tec \& Doc.
}

comme exemple l'entrée "climatique », thème d'un atelier au demeurant bien mené.

En ce qui concerne la première approche, on peut parler de démarche classique : les processus climatiques et hydrologiques sont considérés comme étant du premier ordre, il faut les comprendre, les modéliser et les simuler, et l'on ne peut que limiter ou canaliser leurs effets. La gestion des territoires, avec une préoccupation de gestion de la ressource en eau, est restreinte à des aménagements (ou à des alertes météorologiques). Donc, la démarche est descendante. La dynamique propre du territoire et ses autres déterminants ne sont que peu considérés, car supposés (ou posés) «du second ordre». On peut dire que ce type de démarche est caractéristique des disciplines relevant des sciences de l'univers et non pas, comme on le dit souvent, d'ingénieurs (ceux-ci sont plus circonspects). En fait, la notion de territoire n'est pas vraiment intégrée ; ce qui sert de référence ressort plutôt de la notion de «surface continentale».

Dans la démarche que l'on qualifiera de «non classique », par opposition à la première, on essaye d'abord de comprendre comment fonctionne un territoire. Le climat n'est qu'un des facteurs de ce fonctionnement, même en ce qui concerne la ressource en eau. D'autres facteurs sont considérés comme étant du même ordre (par exemple, l'utilisation des espaces, les usages de la ressource, les composantes écologiques, sociales, politiques et économiques du territoire). Ainsi, les modifications des politiques agricoles ont certainement plus d'effets que le climat sur l'utilisation des terres et, donc, sur la ressource en eau. Un territoire est vu comme un système ayant une dynamique propre, largement autonome, soumis à des « actions extérieures », dont un «forçage » climatique. Il faut comprendre le mieux possible cette dynamique pour le gérer raisonnablement, en particulier pour éviter les catastrophes : celles qui sont dues aux facteurs naturels de forçage, bien sûr, mais aussi celles qui relèvent des... erreurs de gestion. C'est donc une approche systémique.

C'est là où émergent les difficultés : 1'intitulé même du colloque, «Eau et territoires », met au centre le concept de territoire ; il privilégie donc plutôt la seconde démarche et entre en tension avec la première, alors que celle-ci devrait être incluse dans la seconde. Une autre source d'incompréhension découle de la sacro-sainte notion de "processus ». Dans la première démarche, un processus est physique. Dans la deuxième, il peut être aussi écologique (avec les propriétés de la matière vivante) ou anthropique (avec toutes les dimensions liées à l'homme en société). Un autre point de divergence est relatif à la conception même du « système » qu'intègre la notion de territoire; elle implique une conséquence importante, puisqu'il est posé qu'un tel système a une dynamique propre, certes sous influence de facteurs externes, mais, comme on l'a déjà souligné, avec une large part d'autonomie (dès l'échelle régionale). Enfin, les recommandations du rapport du GIEC qui a 
été remis aux divers gouvernements courant juin 2006, et qui insiste sur la nécessité de donner la priorité aux approches actives en matière de stockage de carbone, de «mitigation » et d'adaptation aux changements, devraient plutôt inciter à choisir la deuxième démarche.

On peut donc s'interroger sur les raisons des tenants de la première approche. S'agit-il d'un blocage conceptuel ou cela traduit-il une volonté de défense d'intérêts disciplinaires? Il est difficile de se faire une opinion tranchée. Sans doute est-ce un peu des deux. Inversement, on pourrait aussi critiquer la volonté de tout «socialiser » et, ainsi, de reléguer en arrière-plan les aspects bioécologiques et physicochimiques ou plus simplement « environnementaux », comme cela arrive fréquemment dans les sciences de l'homme et de la société. Là, nous sommes au cœur des enjeux de l'interdisciplinarité.

On pourrait résumer le "scénario » du thème proposé à la discussion par quelques mots-clés : une ressource, des risques, des écosystèmes, des aménagements et des sociétés humaines. Mais c'est un peu bref. On pourrait dire aussi que ce colloque a incité à réfléchir plus largement sur le contour scientifique de ce que pourraient être des « sciences de l'environnement et du développement (durable) » regroupant des compétences provenant des secteurs traditionnels. C'est d'un grand intérêt, mais $c^{\prime}$ est encore un peu trop général dans ce contexte et cela concerne surtout nos institutions de recherche.

En fait, plus précisément, le colloque nous a montré, aussi bien en termes scientifiques qu'en termes de gestion et d'ingénierie, qu'il faut considérer un ensemble intégrant : (1) disponibilité et variabilité, (2) réseau hydrographique, (3) usages multiples et diversité des acteurs impliqués, (4) aménagement et (5) risques associés, en le situant dans (6) un espace géographique, assujetti à une contrainte économique et plongé dans un contexte politique. Comme la notion de territoire recouvre précisément les points (2), (3), (4), (5) et (6), on comprend bien que la question de la ressource et de sa gestion n'a de sens que par rapport à cette notion centrale de territoire. Tout n'est donc pas réductible aux «flux naturels »; on pourrait même pousser le paradoxe jusqu'à dire que c'est du second ordre. Ainsi, pour pallier les deficits locaux en eau, les Carthaginois, puis les Romains, ont construit un aqueduc de $132 \mathrm{~km}$ pour amener l'eau dans Carthage et dans les champs qui l'entouraient. Les exemples actuels, de ce type, foisonnent.

Une fois cela dit, tout reste à faire... Il reste notamment à mettre la communauté scientifique en ordre de bataille pour appréhender de façon intégrée, systémique, multiéchelle et interdisciplinaire ce problème; et c'est loin d'être fait. Point à souligner : dans cet effort, il convient de veiller à ne pas sacrifier à l'utilitarisme immédiat, mais au contraire de satisfaire à l'impérieuse nécessité d'une réflexion conceptuelle. Dans le contexte actuel, on n'insiste pas suffisamment sur ce dernier aspect, pourtant sous la responsabilité première de la communauté scientifique : il ne faut pas seulement acquérir données et connaissances, mais aussi les organiser, puis en tirer des notions et des outils génériques et, pour cela, conceptualiser. C'est à ce prix que nous obtenons un support scientifique efficace pour la science elle-même, mais aussi et surtout pour sa traduction en termes d'ingénierie, de gestion et de décision.

Comme le propose le Cemagref, il s'agit, aujourd'hui, de concevoir un vrai programme interdisciplinaire intégrant les sciences de la nature (hydrologie, pédologie, écologie), les sciences de l'homme et de la société (sociologie, géographie, économie, histoire), ainsi que - et peut-être surtout - les sciences de l'ingénieur (pour les aménagements, les diverses régulations associées, mais aussi pour les agrosystèmes et, dans l'avenir, pour les écosystèmes). Il s'agit également de promouvoir et de développer les méthodologies et les techniques nécessaires à cette recherche et à sa finalisation, notamment en termes d'ingénierie et de gestion. Bien sûr, l'exercice est difficile, le risque est grand de se perdre dans le brouillard de l'approximatif, mais, là encore, le texte de cadrage proposé traçait des pistes efficaces. Les conclusions qui ont été tirées du colloque, bien plus détaillées que ce qui est présenté ici, constituent une réelle avancée. On peut même dire que ce colloque a été un événement dont on n'a pas encore pris la juste mesure et qu'il reste encore à en exploiter tous les acquis. On est rassuré quand on sait que le ministère de l'Écologie et du Développement durable de l'époque, le département Environnement et développement durable du CNRS et, bien sûr, le Cemagref, ont décidé de lancer une action commune sur le sujet en 2007. À quand l'implication d'autres partenaires? 